\title{
An age-related shift of resting-state functional connectivity of the subthalamic nucleus: a potential mechanism for compensating motor performance decline in older adults
}

\section{Christian Mathys ${ }^{1}$, Felix Hoffstaedter ${ }^{2,3}$, Julian Caspers ${ }^{1,3}$, Svenja Caspers ${ }^{3}$, Martin Südmeyer ${ }^{2,4}$, Christian Grefkes ${ }^{3,5,6}$, Simon B. Eickhoff ${ }^{2,3}$ and Robert Langner ${ }^{2,3} *$}

\author{
${ }^{1}$ Department of Diagnostic and Interventional Radiology, Medical Faculty, Heinrich Heine University Düsseldorf, Düsseldorf, Germany \\ 2 Institute of Clinical Neuroscience and Medical Psychology, Medical Faculty, Heinrich Heine University Düsseldorf, Düsseldorf, Germany \\ ${ }^{3}$ Institute of Neuroscience and Medicine (INM-1, INM-3), Research Centre Jülich, Jülich, Germany \\ ${ }^{4}$ Center for Movement Disorders and Neuromodulation, Department of Neurology, Medical Faculty, Heinrich Heine University Düsseldorf, Düsseldorf, Germany \\ ${ }^{5}$ Neuromodulation and Neurorehabilitation Group, Max Planck Institute for Neurological Research, Cologne, Germany \\ ${ }^{6}$ Department of Neurology, University of Cologne, Cologne, Germany
}

\section{Edited by:}

Agustin Ibanez, Institute of Cognitive Neurology, Argentina

Reviewed by:

Fiorenzo Conti, Universita Politecnica delle Marche, Italy

Aurel Popa-Wagner, Clinic of

Psychiatry, Germany

${ }^{*}$ Correspondence:

Robert Langner, Institute of Clinical Neuroscience and Medical

Psychology, Heinrich Heine University Düsseldorf, Universitätsstr. 1,

D-40225 Düsseldorf, Germany

e-mail: robert.langner@uni-

duesseldorf.de
Healthy aging is associated with decline in basic motor functioning and higher motor control. Here, we investigated age-related differences in the brain-wide functional connectivity (FC) pattern of the subthalamic nucleus (STN), which plays an important role in motor response control. As earlier studies revealed functional coupling between STN and basal ganglia, which both are known to influence the conservativeness of motor responses on a superordinate level, we tested the hypothesis that STN FC with the striatum becomes dysbalanced with age. To this end, we performed a seed-based resting-state analysis of fMRI data from 361 healthy adults (mean age: 41.8, age range: 18-85) using bilateral STN as the seed region of interest. Age was included as a covariate to identify regions showing age-related changes of FC with the STN seed. The analysis revealed positive FC of the STN with several previously described subcortical and cortical regions like the anterior cingulate and sensorimotor cortex, as well as not-yet reported regions including central and posterior insula. With increasing age, we observed reduced positive FC with caudate nucleus, thalamus, and insula as well as increased positive FC with sensorimotor cortex and putamen. Furthermore, an age-related reduction of negative FC was found with precuneus and posterior cingulate cortex. We suggest that this reduced de-coupling of brain areas involved in self-relevant but motor-unrelated cognitive processing (i.e. precuneus and posterior cingulate cortex) from the STN motor network may represent a potential mechanism behind the age-dependent decline in motor performance. At the same time, older adults appear to compensate for this decline by releasing superordinate motor control areas, in particular caudate nucleus and insula, from STN interference while increasing STN-mediated response control over lower level motor areas like sensorimotor cortex and putamen.

Keywords: subthalamic nucleus, resting-state fMRI, functional connectivity, healthy aging, age-related changes

\section{INTRODUCTION}

The proportion of the world's population over 60 years of age will presumably double from about $11-22 \%$ (i.e., amounting to 2 billion people) between the years 2000 and $2050^{1}$. There is thus a need for a more profound comprehension of the psychological and physiological changes associated with healthy aging.

A typical feature of aging is a deterioration of motor control (Seidler et al., 2010), the neural substrates of which have recently moved into the focus of research (Heuninckx et al., 2005; Ward et al., 2008; Harada et al., 2009; Seidler et al., 2010; Noble et al., 2011; Goble et al., 2012). For instance, it was shown that deterioration of motor performance in advanced age is associated with

\footnotetext{
${ }^{1}$ www.who.int/ageing
}

a more widespread involvement of prefrontal cortex and basal ganglia, which might reflect an increased reliance on cognitive control mechanisms, in compensation for lower level sensorimotor processing deficits (Seidler et al., 2010).

A key structure that is connected to both motor system and cognitive areas is the subthalamic nucleus (STN, Hamani et al., 2004; Temel etal., 2005). The STN is an integral part of current models of basal ganglia motor functions, which differentiate direct and indirect striatal output pathways, originating from different neuronal populations and which are characterized by opposite end-effects (Weintraub and Zaghloul, 2013). The direct pathway represents inhibitory circuits from the striatum to the internal segment of the globus pallidus, leading to a decreased inhibition of the ventrolateral thalamic nucleus, which in turn is associated with motor facilitation (Weintraub and Zaghloul, 
2013). In contrast, the STN is a key structure of the polysynaptic indirect pathway. In addition to inhibitory striatal afferents, the internal segment of the globus pallidus is stimulated by the STN, which then leads to motor inhibition via an increased inhibition of the ventrolateral thalamus (Alexander and Crutcher, 1990). Both STN and striatum receive excitatory input from primary motor cortex (M1), premotor cortex (PMC), and primary somatosensory cortical (S1) areas (Weintraub and Zaghloul, 2013). Previous studies on STN functional connectivity (FC) based on resting-state (RS) functional magnetic resonance imaging (fMRI; Baudrexel et al., 2011; Brunenberg et al., 2012) as well as on its anatomical connectivity using diffusion-weighted imaging (DWI; Lambert et al., 2012) showed bilateral coupling with the striatum including caudate nucleus, putamen, and globus pallidus, as well as midbrain, thalamus, dorsal pontine areas, cerebellum, supplementary motor area (SMA) cortex, and lateral PMC. The STN's involvement in motor functions is further corroborated by marked improvements in motor functioning after electrical stimulation of the STN in Parkinson's disease (PD; Limousin et al., 1995).

Key functions of the STN in the context of motor control include the control of response thresholds in the fronto-striatal network (Mansfield et al., 2011), thereby playing a role in stopping of ongoing motor activity (Aron et al., 2007) and inhibiting responses during conflict (Frank et al., 2007; Brittain et al., 2012). According to recent models, competing cortical input from a "hyperdirect pathway" (excitatory projection from PMC and M1 to STN) is integrated in the STN to dynamically adjust the response threshold (Nambu et al., 2002; Frank, 2006). It is important to appreciate, however, that the STN has not only been implicated in motor control but has also been related to affective and cognitive processes, given that it is connected to key regions of these systems (Hamani et al., 2004; Temel et al., 2005). Thus, the STN seems to be one of the gateways between motor control and cognition.

Given age-related changes in cognitive and motor performance as well as their integration (for a review, see Seidler et al., 2010), the location of the STN at the interface of these networks raises the question of how this STN network may change in the course of healthy aging. Besides changes in regional brain structure and activity, differences in interregional coupling patterns have been increasingly recognized as a neural substrate of behavioral changes with age (Lee et al., 2006; Madden et al., 2010; Langner et al., 2014). Such age-related FC changes have shown to occur independently from structural changes (Hoffstaedter et al., 2014; Langner et al., 2014) and to predict behavioral changes, even in the absence of changes in regional activation (Grady, 2005; Madden et al., 2010). Therefore, in this study, we aimed to examine age-related changes of STN FC. Given previous evidence for increased reliance on cognitive control mechanisms in the context of age-related deficits in lower level motor control (Seidler et al., 2010), we expect a shift in functional integration of the STN with the striatum in the elderly, since both regions are known to influence the cognitively mediated conservativeness of motor responses (Mansfield et al., 2011). We further hypothesized that RS FC of the STN with the striatum becomes dysbalanced with age.

\section{MATERIALS AND METHODS SEED DEFINITION}

Bilateral STN seed regions of interest (ROIs; see Figure 1) were derived from a recently published atlas that was created using high-resolution susceptibility mapping of this brain area in eight healthy individuals using 7-T MRI (Schafer et al., 2012). Previous studies have reported a slight shift of the STN in lateral direction with increasing age (Keuken et al., 2013). However, we consider this shift irrelevant for the present study because the extent of the Gaussian kernel we used for spatial smoothing $(5 \mathrm{~mm})$ clearly exceeded the size of the observed shift $(\approx 2 \mathrm{~mm})$. For the main network analysis, the activation time series (first eigenvariate) of both left and right STN ROIs were collapsed into a common seed to increase the reliability of the analysis. In a supplementary analysis of hemispheric differences, the two ROIs were used as separate seeds.

\section{SAMPLE}

The sample included RS fMRI data from 361 healthy adults $(34.5 \%$ \% $)$ with a mean age of $41.9(\mathrm{SD}=16.5$, median $=41$, interquartile range $=28$ ) years. All volunteers gave written informed consent to the study and were without any record of

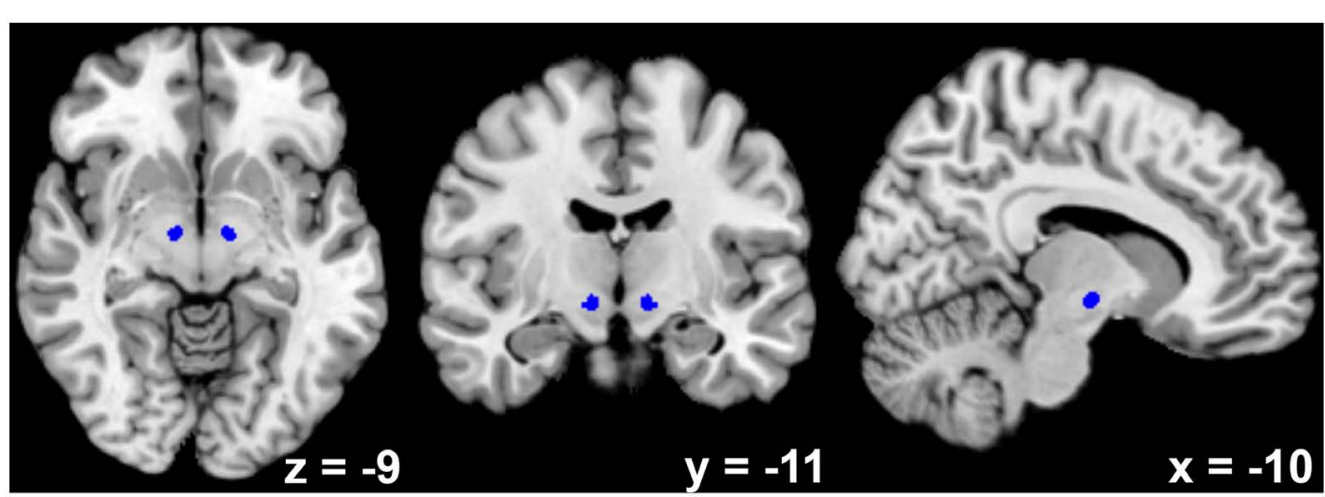

FIGURE 1 | Depiction of the bilateral subthalamic nucleus seed region as derived from high-resolution susceptibility mapping using 7-T magnetic resonance imaging (Schafer et al., 2012). 
Table 1 | Characteristics of the sample.

\begin{tabular}{lllll}
\hline Contribution Site & $\boldsymbol{n}$ & Mean age (range) & Sex: male (\%) & Measurement parameters $^{\mathbf{a}}$ \\
\hline RWTH University Hospital Aachen, Germany & 47 & $36.5(19-59)$ & 46 & $3 \mathrm{~T} / 250 / 2.2 / 30 / 80^{\circ} / 3.1 \mathrm{~mm} \times 3.1 \mathrm{~mm} \times 3.1 \mathrm{~mm}$ \\
& 28 & $63.4(55-72)$ & 71 & $3 \mathrm{~T} / 270 / 2.2 / 30 / 90^{\circ} / 3.1 \mathrm{~mm} \times 3.1 \mathrm{~mm} \times 3.1 \mathrm{~mm}$ \\
Research Centre Jülich, Germany & 54 & $28.5(18-59)$ & 54 & $3 \mathrm{~T} / 250 / 2.2 / 30 / 90^{\circ} / 3.1 \mathrm{~mm} \times 3.1 \mathrm{~mm} \times 3.1 \mathrm{~mm}$ \\
NKI/Rockland, Orangeburg, NY, USA & 100 & $45.1(21-71)$ & 49 & $3 \mathrm{~T} / 300 / 2.2 / 30 / 90^{\circ} / 3.1 \mathrm{~mm} \times 3.1 \mathrm{~mm} \times 3.1 \mathrm{~mm}$ \\
\hline
\end{tabular}

NKI, Nathan S. Kline Institute.

a Measurement parameters: magnetic field strength of the scanner/number of acquired volumes/repetition time (in s)/echo time (in ms)/flip angle/voxel size.

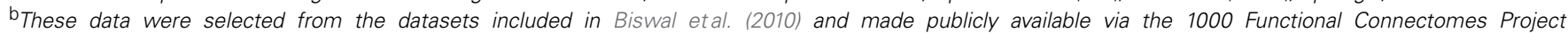
http://fcon_1000.projects.nitrc.org.

${ }^{c}$ All but the participants of the NKI/Rockland sample were instructed to keep their eyes closed during the measurement.

neurological or psychiatric disorder. Three different sites contributed data (see Table 1). Approval for the joint (re-)analysis was obtained from the local ethics committee of the University Hospital Düsseldorf.

\section{DATA ACOUISITION AND PREPROCESSING}

Echo-planar imaging (EPI) of the entire brain (vertex to lower parts of the cerebellum) at $3 \mathrm{~T}$ was used to record $>9 \mathrm{~min}$ of blood oxygen level-dependent (BOLD) activity in all participants (detailed measurement parameters are provided in Table 1). The scan parameters of the three different sites were not identical but similar (see Table 1). Site effects were also removed in the group-level data analysis (see below). Participants were instructed to let their mind wander and not to fall asleep. Joint preprocessing of all data was performed with $\mathrm{SPM} 8^{2}$. The first four images of each BOLD time series were discarded to account for saturation effects. A two-pass affine registration procedure was used for motion correction. The images were initially realigned to the first image and afterwards to the mean of the realigned images. The mean image was also used for normalization to the Montreal Neurological Institute (MNI) single-subject template as provided in SPM8, using the unified segmentation approach (Ashburner and Friston, 2005). The same normalization parameters were then applied to each individual EPI volume. During normalization, image volumes were resampled to a voxel size of $1.5 \mathrm{~mm} \times 1.5 \mathrm{~mm} \times 1.5 \mathrm{~mm}$. A 5-mm full-width at half-maximum Gaussian kernel was subsequently applied to spatially smooth the images.

\section{DATA ANALYSIS}

We used a validated protocol (Satterthwaite et al., 2013b) that has been proven to reliably detect age-related FC-changes, which is especially important in the context of known correlations between age and in-scanner motion. Accordingly, to avoid correlations explained by nuisance variables, variance explained by the following parameters was removed (Jakobs et al., 2012; Satterthwaite et al., 2013a): (i) the six motion parameters obtained from spatial realignment, (ii) the first derivates of the six motion parameters, (iii) the time series of the gray-matter, white-matter, and cerebrospinal-fluid signal intensity obtained by averaging, at each

\footnotetext{
${ }^{2}$ www.fil.ion.ucl.ac.uk/spm
}

point in time, across all voxels of the respective tissue class (as determined by the SPM8 segmentation). In order to remove residual low- and high-frequency noise, the data were band-pass filtered preserving frequencies between 0.01 and $0.08 \mathrm{~Hz}$ (Biswal et al., 1995; Greicius et al., 2003; Fox and Raichle, 2007).

The time series of both STNs, represented by the first eigenvariate of all STN voxels' time series, was then correlated with the time series of all other gray-matter voxels, and the resulting Pearson correlation coefficients were transformed into Fisher's $Z$ scores for every voxel. In the subsequent group-level analysis of variance (ANOVA), sex, data contribution site (three subsamples), and age were used as covariates.

Age effects on STN FC were tested in conjunction with the respective FC main effect. For example, age-related decreases of positive FC were tested using the following conjunction: [positive FC with bilateral STN] $\cap$ [age-related decrease of FC with bilateral STN]. For the supplementary analysis of hemispheric differences between left and right STN, the same approach was adopted. Results were considered significant at cluster-level $p<0.05$ (family-wise error-corrected for multiple comparisons; clusterforming threshold at voxel level: $p<0.001$ ). For exploratory purposes, a more liberal cluster-level threshold of $p<0.1$ was also adopted for the analysis of age-dependent decline in positive FC of STN. Resulting brain areas were anatomically assigned via the SPM Anatomy toolbox ${ }^{3}$ (Eickhoff et al., 2007), which implemented probabilistic cytoarchitectonic mapping (Zilles and Amunts, 2010).

A split-half group comparison between the 180 younger und 180 older participants was also performed. Clusters for age effects and hemispheric differences are only reported if their time series show a correlation of at least $r>0.1$ or $r<-0.1$ (equivalent to at least a small effect size according to Cohen's definition, Cohen, 1988) with the STN time series for either the 180 younger or the 180 older participants or both.

\section{RESULTS}

\section{WHOLE-BRAIN FC PATTERN OF THE STN}

Bilateral STN RS-FC analysis revealed a significantly positive coupling between STN and the following areas bilaterally

\footnotetext{
${ }^{3}$ http://www.fz-juelich.de/ime/spm_anatomy_toolbox, V1.8
} 


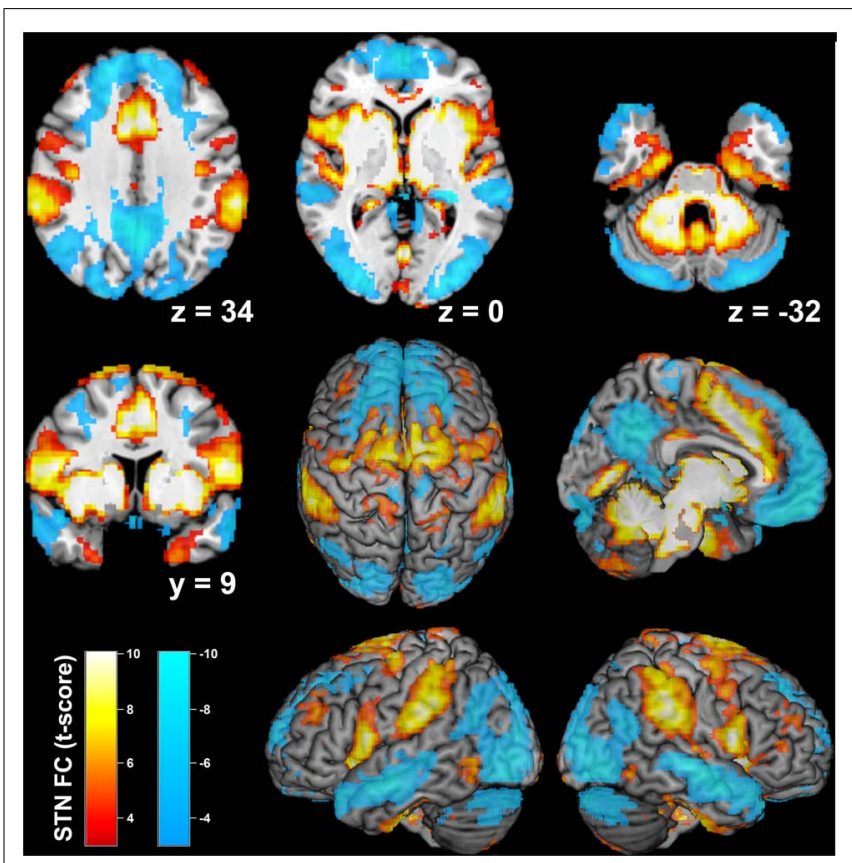

FIGURE 2 | Resting-state functional connectivity of the bilateral subthalamic nucleus seed region. Positive network coupling is denoted in warm colors, while cold colors denote anticorrelated regions. Montreal Neurological Institute coordinates are provided for transsectional slices. Surface rendering was performed with MRIcron (Rorden and Brett, 2000).

(Figure 2): putamen, caudate nucleus, external and internal globus pallidus (GPe and GPi), thalamus (prefrontal, temporal, parietal, premotor, somatosensory and motor. Behrens et al., 2003), anterior and midcingulate cortex, posterior (Kurth et al., 2010a) and central insula extending to the parietal operculum (areas
OP 1-4, Eickhoff et al., 2006a,b), inferior frontal gyrus (areas 44/45, Amunts etal., 1999, 2004), dorsolateral prefrontal cortex, PMC (area 6, Geyer, 2004), S1 and M1 (areas 1, 2, 3a, 3b, and 4, Geyer et al., 1996, 1999, 2000; Grefkes et al., 2001; Geyer, 2004), rostral superior parietal cortex (area 5Ci, Scheperjans et al., 2008a,b), rostral inferior parietal cortex (PF, PFm, PFt, PFop, PFcm, Caspers et al., 2006, 2008), primary visual cortex (V1; area 17, Amunts et al., 2000), hippocampus (area EC, Amunts et al., 2005), cerebellum (lobules I-VI, Diedrichsen et al., 2009), midbrain, and pons.

Anticorrelated regions (Figure 2) comprised bilateral orbitofrontal cortex, PMC and M1 (area 4, Geyer et al., 1996), posterior cingulate cortex, precuneus, posterior S1 (area 2, Grefkes et al., 2001), caudal superior parietal cortex (area 7a, Scheperjans et al., 2008a,b), caudal inferior parietal lobule (PGa, Caspers et al., 2006, 2008), V1 and secondary visual cortex (Amunts et al., 2000), cerebellum (lobules VIIa, IX, Diedrichsen et al., 2009). Anticorrelated regions were also observed in bilateral hippocampus (area CA, SUB, and FD Amunts et al., 2005) and amygdala (area LB and SF, Amunts et al., 2005). Interestingly, different fractions of M1, PMC, S1, and V1 showed positive or negative FC with STN, respectively (Figure $\mathbf{3}$ ).

In the PMC and SMA, we found a considerably larger proportion of voxels with positive than negative FC with the seed (Figure 3). While positively correlated voxels were distributed across the whole PMC and SMA regions, negatively correlated voxels were mostly located medially at the anterior and posterior border of the SMA. In M1, we found moderately more negatively correlated voxels (Figure 3), which were predominantly found medially in the anterior part of Area 4 (Area 4a, Geyer et al., 1996), while positively correlated clusters were found laterally and medially in its posterior part (Area 4p, Geyer et al., 1996).

In the posterior $\mathrm{S} 1$, we found moderately more voxels positively correlated with STN; they were more numerous in the lateral part,

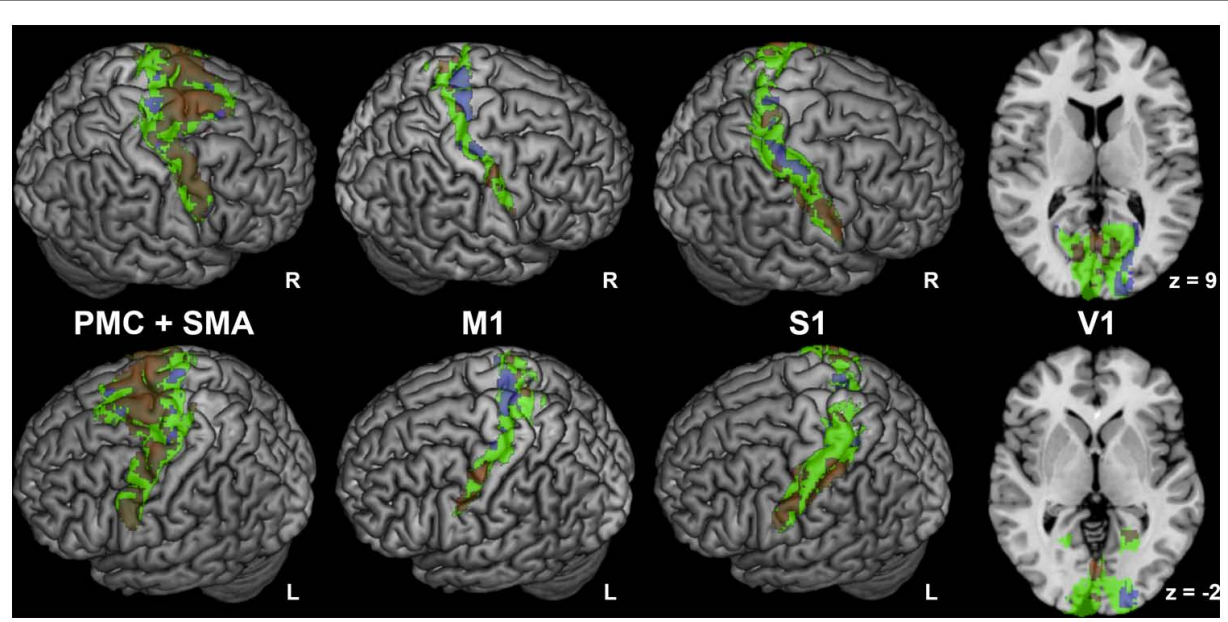

FIGURE 3 | Regions with mixed connectivity pattern to subthalamic nucleus. Different fractions of premotor corex and supplementary motor area (PMC and SMA; Geyer, 2004), primary motor cortex (M1; Geyer et al., 1996), primary sensory cortex (S1; Geyer etal., 1999, 2000; Grefkes et al., 2001) and primary visual cortex (V1; Amunts et al., 2000) showing positive or negative functional connectivity with the subthalamic nucleus. Cytoarchitectonic regions are denoted in green, positive network coupling in warm colors, and anticorrelated network coupling in cold colors. Correlated and anticorrelated clusters do not overlap; this impression is an artefactual result of the surface rendering of 3-D clusters. 
Table $\mathbf{2}$ | Adult age differences in intrinsic functional connectivity between both STNs and associated brain regions.

\begin{tabular}{|c|c|c|c|c|c|}
\hline Pair of regions & Coordinates $x / y / z(\mathrm{~mm})$ & Mean $r_{\text {young }}$ & Mean $r_{\text {elderly }}$ & $T$-score $\Delta r$ & Corr. with age $\left(r_{s}\right)$ \\
\hline L STN-R STN & N/A & 0.388 & 0.474 & -1.82 & 0.17 \\
\hline STN-R posterior insula & $38 /-18 / 6$ & 0.152 & 0.001 & $2.58^{*}$ & -0.24 \\
\hline STN-R caudate nucleus/thalamus & $16 /-6 / 20$ & 0.138 & -0.004 & $3.08 * *$ & -0.23 \\
\hline STN-R sensorimotor & $42 /-14 / 34$ & 0.028 & 0.141 & $-4.44 * * *$ & 0.28 \\
\hline STN-L precuneus/PCC & $-8 /-56 / 42$ & -0.107 & 0.011 & $-5.33^{* * *}$ & 0.28 \\
\hline STN-R V3/N4 & $34 /-83 /-12$ & -0.037 & -0.119 & 1.90 & -0.23 \\
\hline
\end{tabular}

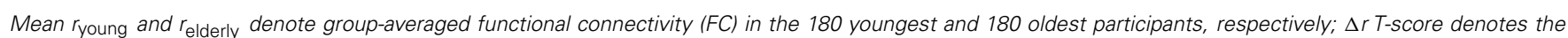
T-statistic $\left({ }^{*} p<0.05 ;{ }^{*} p<0.01 ;{ }^{* *} p<0.001\right)$ for the comparison of the two FC-values between both subgroups.

while anticorrelated voxels were mostly located medially and were more pronounced on the right side (see Figure 3).

In V1, voxels showing positive FC with STN were located bilaterally in the rostral part, while anticorrelated voxels were nearly exclusively found in the right-sided occipital part of this region.

\section{AGE EFFECTS ON THE FC PATTERN OF THE STN}

There were several differences in RS-FC related to age (see Table 2): Age-dependent decline (Figure 4) in positive FC of STN was found within the caudate nucleus of the right striatum, right thalamus (prefrontal and parietal zone, Behrens et al., 2003), and right central and posterior insula (Ig2, Kurth et al., 2010a). There was a trend toward significance for a cluster within the left central insula $(p=0.072)$.

Increases in positive STN FC with age were found for right sensorimotor cortex (areas 3a, 4p) and left putamen (see Figure 5).

Age-related decrease in negative STN FC (i.e., diminished anti-correlation with age; see Figure 6) was detected for left precuneus extending to posterior cingulate cortex, while stronger anti-correlation with age (see Table 2) was observed for right ventral extrastriate cortex (area V3/V4, Rottschy et al., 2007).

\section{SUPPLEMENTARY ANALYSIS OF HEMISPHERIC DIFFERENCES IN AGE-RELATED FC CHANGES}

Positive FC between left and right STN increased significantly with age (Figure 7, Table 2). There were no clusters in the brain that showed a significantly stronger positive or negative FC with either the left or right STN. Also, no hemispheric differences were found for the eight possible combinations of age-related increase/decline, positive/negative FC, and preponderance for left/right STN.

\section{DISCUSSION}

In this RS fMRI study in 361 participants an atlas-based analysis (SPM Anatomy toolbox V1.8 with implemented probabilistic cytoarchitectonic mapping, Eickhoff et al., 2007; Zilles and Amunts, 2010) revealed complex connectivity patterns, with positive and negative FC to the STN for the PMC, M1, S1, and V1. Moreover, we found several age-related changes of FC with STN. According to our hypothesis, the STN's FC with the caudate nucleus and also with the central insula declined with age, but at the same time increased with the putamen and also right M1. Notably, anti-correlations with STN decreased with age for the precuneus and left posterior cingulate cortex, while they increased with age for right ventral extrastriate cortex.

\section{FUNCTIONAL CONNECTIVITY OF THE STN}

The pattern of RS-FC of bilateral STN in our sample is largely in line with previous reports on STN connectivity, comprising RSFC analysis, DWI-based studies, tract tracing studies in animals and positron emission tomography (PET) studies (Aravamuthan et al., 2007; Le Jeune et al., 2010; Baudrexel et al., 2011; Brunenberg et al., 2012; Lambert et al., 2012; Manes et al., 2013). After deep brain stimulation (DBS) of the STN in Parkinson patients, PET studies revealed decreased glucose metabolism in brain areas that were reported to show positive coupling with STN (Le Jeune et al., 2010). This was in line with the observation that high-frequency stimulation of the STN reduces firing rate and oscillatory activity of STN neurons and in the STN network (Meissner et al., 2005).

The presence of FC between the STN and basal ganglia as well as thalamus is in strong concordance with current models of basal ganglia anatomy and physiology (see Introduction). Also, the activation of anterior and midcingulate cortex has repeatedly been associated with attention and related motor control functions, such as output-related attention, preparation, and response selection (Devinsky et al., 1995; Jueptner et al., 1997; Rubia et al., 1999; Langner et al., 2011, 2012; Cieslik et al., 2013; Hoffstaedter et al., 2013a,b). In concordance with these functions, connectivity between cingulate cortex and basal ganglia has been previously described (Margulies et al., 2007; Beckmann et al., 2009; Kelly et al., 2009; Hoffstaedter et al., 2013a). The inclusion of the STN in this network appears reasonable, because the aforementioned cognitive functions involve the inhibition of certain responses in favor of others, which is one of the presumed functions of the STN (Frank et al., 2007; Brittain et al., 2012).

Besides positive FC, we found functional anticorrelations (i.e., neural de-coupling) between STN and posterior cingulate cortex and precuneus. This agrees well with previous reports on the FC pattern of the STN (Baudrexel et al., 2011). DWI studies also suggested a significant structural connectivity of STN and posterior cingulate gyrus (Brunenberg et al., 2012; Lambert et al., 2012). 

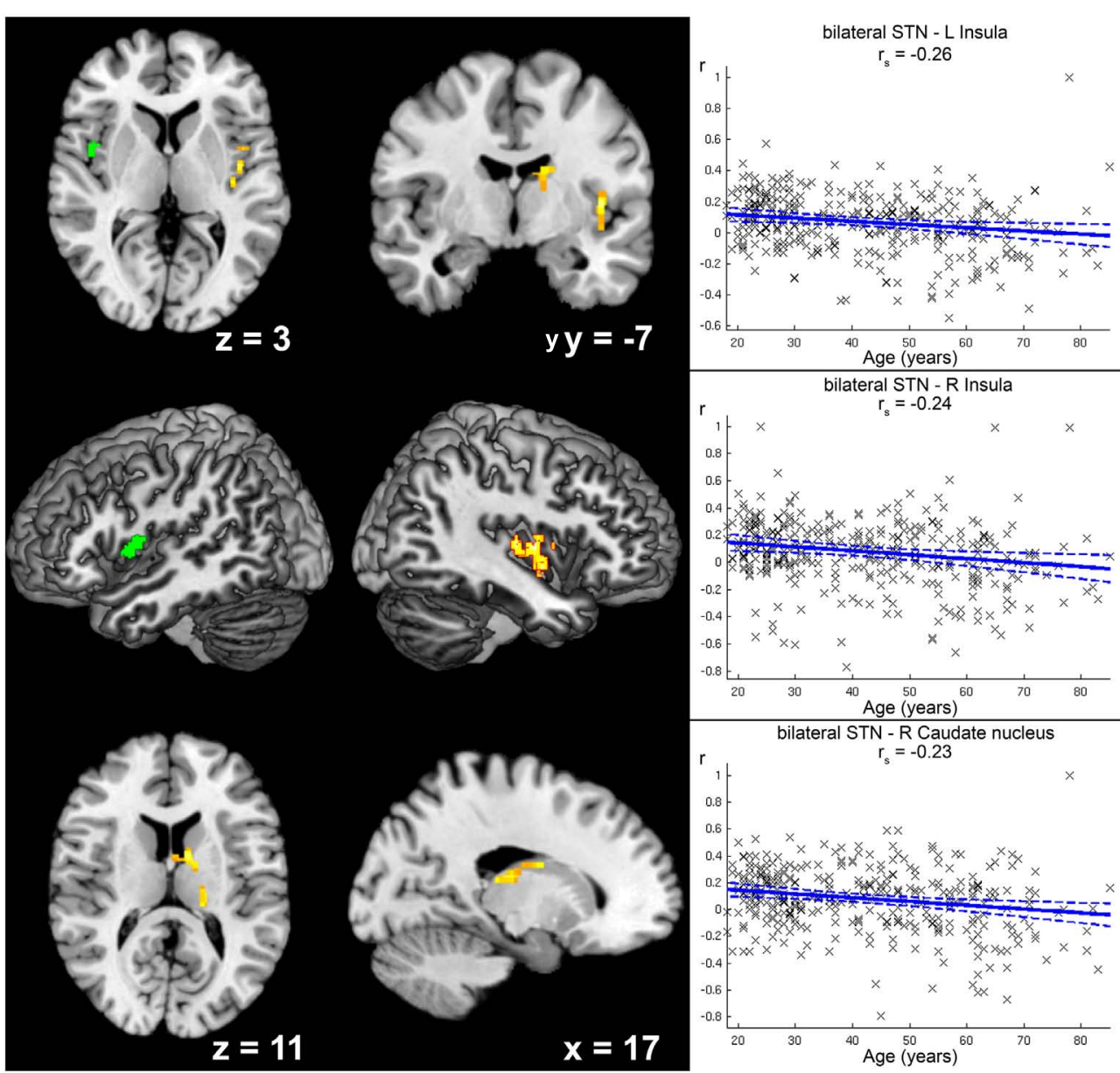

FIGURE 4 | Age-related decrease in positive functional connectivity (FC) with the subthalamic nucleus (STN). Clusters showing a significant age-related decrease in positive functional FC with the seed region (i.e., bilateral STN) are marked in warm colors, while the green cluster was only marginally significant ( $p=0.072)$ after correction for multiple testing. Coordinates refer to Montreal Neurological Institute space. Surface reconstructions of both hemispheres were cropped to allow visibility of insular clusters. Scatter plots for each of the three clusters show the individual FC values against age across all participants. Linear regression lines with 95\% confidence interval (dotted lines), as well as $r_{\mathrm{S}}$-values (Spearman's rank correlation coefficient) are provided for each significant cluster, respectively.
Along the same lines, PET scans after STN inhibition by DBS showed an increased glucose metabolism for posterior cingulate gyrus on both sides (Le Jeune et al., 2010), which is consistent with the observed functional anticorrelation. Since posterior cingulate cortex and precuneus are essentially related to non-motor functions (e.g. introspection, as discussed in more detail below), de-coupling of these areas from the STN's motor network appears very reasonable. As for any non-motor cognitive functions of the STN, it seems that the networks involved in these functions are most likely not coupled to the posterior cingulate cortex and precuneus, which are also known to be part of the default-mode network (Leech and Sharp, 2013).

Some regions showed a mixed connectivity pattern, with different subregions showing positive or negative FC with STN. PMC and SMA were mostly positively correlated with the STN. Although clear distinction from neighboring regions (especially M1) is difficult, some anticorrelated clusters were found in the SMA. It is unclear whether the STN drives PMC and SMA or, vice versa, the
STN is driven by these regions. However, in case of a direct influence from STN on PMC and SMA, pathological hyperactivity of the STN in PD patients could interfere with most of both areas, but parts of the SMA region appear to be excluded from this interference and may thus have higher chances to be part of compensation mechanisms. Correspondingly, recent reports of increased SMAM1 coherence in magnetoencephalography data of PD patients have been interpreted as a compensatory mechanism (Pollok et al., 2013).

In M1, positively correlated voxels accumulated laterally at the face representation (Meier et al., 2008). Correspondingly, reduction of glucose metabolism in PET scans after STN inhibition by DBS was more pronounced in the lateral aspects of M1 (Le Jeune et al., 2010). To the best of our knowledge, there is no further evidence in the literature for the possible conclusion that the STN's control over facial motor functions could be stronger than over extremity functions. On the other hand, the STN's particular functional connection to facial motoneurons might represent 

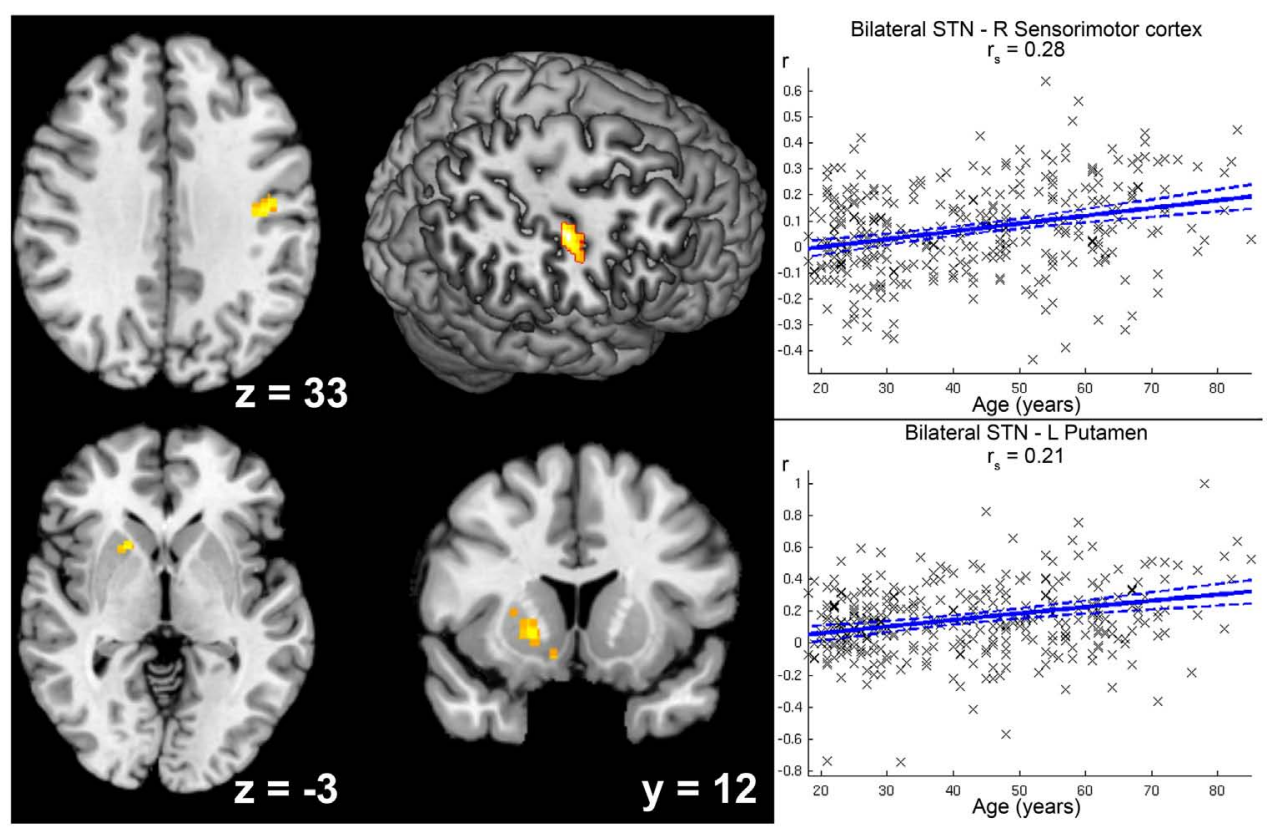

FIGURE 5 | Age-related increase in positive functional connectivity (FC) with the subthalamic nucleus (STN). Clusters showing a significant age-related increase in positive FC with the seed region (i.e., bilateral STN) are marked in warm colors. Coordinates refer to Montreal Neurological Institute space. The second image in the first row shows a right-sided ascending $\left(45^{\circ}\right)$ view on a surface reconstruction of the brain.
Parts of the right central region have been cropped away to allow visibility of the cluster in right sensorimotor cortex. Scatter plots for each of the two clusters show the individual $\mathrm{FC}$ values against age across all participants. Linear regression lines with 95\% confidence interval (dotted lines), as well as $r_{\mathrm{S}}$-values (Spearman's rank correlation coefficient) are provided for each significant cluster, respectively. a substrate for apraxia of lid opening, which is a typical side effect after DBS in PD patients (Tommasi et al., 2012) and might indicate co-stimulation and consecutive dysfunction of STN-connected regions.

Within the STN's elaborate functional network of correlated and anticorrelated brain areas, we found several age-related changes, which will be discussed in the following section.

\section{AGE-RELATED CHANGES OF POSITIVE FC OF THE STN}

According to our hypothesis, the STN's positive FC with the striatum declined with age in the caudate nucleus and also in the insula, but at the same time, increased with age in the putamen and also in $\mathrm{M} 1$.

According to a modular conception of the striatum, the caudate nucleus is thought to contribute to cognitive functions like goal-directed action selection by evaluation of action-outcomes, while the putamen appears to be more limited to sensorimotor coordination (Grahn et al., 2008).

Involvement of the caudate nucleus in cognitive functions is supported by several studies, which found this brain area to be an important node in learning, memory, and feedback-processing (Packard and Knowlton, 2002; Graybiel, 2005). Also, microstimulation of the caudate nucleus has been shown to enhance associative learning in primates (Williams and Eskandar, 2006).

On the other hand, a recent activation likelihood estimation (ALE) meta-analysis across 35 sensorimotor tasks revealed consistent bilateral activation in the putamen (Hardwick et al., 2013). The putamen is thought to synchronize cortical activity that is associated with the selection and propagation of movements (Brown and Marsden, 1998). Specifically, at the initiation of bimanual movements, the putamen showed peak activations bilaterally, which was interpreted as the point in time when specific contributions from higher and lower level cortical motor areas have to be synchronized (Kraft et al., 2007).

By transferring this concept to our results, we observe an agerelated shift of the STN coupling away from cognitive-motor (action selection; caudate nucleus) toward basic-motor functions (sensorimotor coordination; putamen). The STN is believed to play a role in motor inhibition and, particularly, in response inhibition and the setting of response thresholds (Aron et al., 2007; Mansfield et al., 2011). Since deterioration of motor control is well known in advanced age (Seidler et al., 2010), a stronger inhibitory influence of the STN on the putamen's executive motor functions toward more response conservativeness appears reasonable. Previous evidence also points to an increased reliance on cognitive control mechanisms in the context of age-related deficits in lower level motor control (Seidler et al., 2010). Apparently, one aspect of this adjustment process is to release the caudate nucleus from interference by the STN. This might allow a more careful selection of feasible actions by the caudate nucleus.

Age-related changes found in thalamus, insula, and sensorimotor cortex agree well with this concept. In the thalamus, we observed an age-dependent decline of positive FC with STN. The affected prefrontal subregion of the thalamus is involved in cognitive processing like attention, planning, organization, abstract thinking, active memory, and multi-tasking (Eckert et al., 2012). 


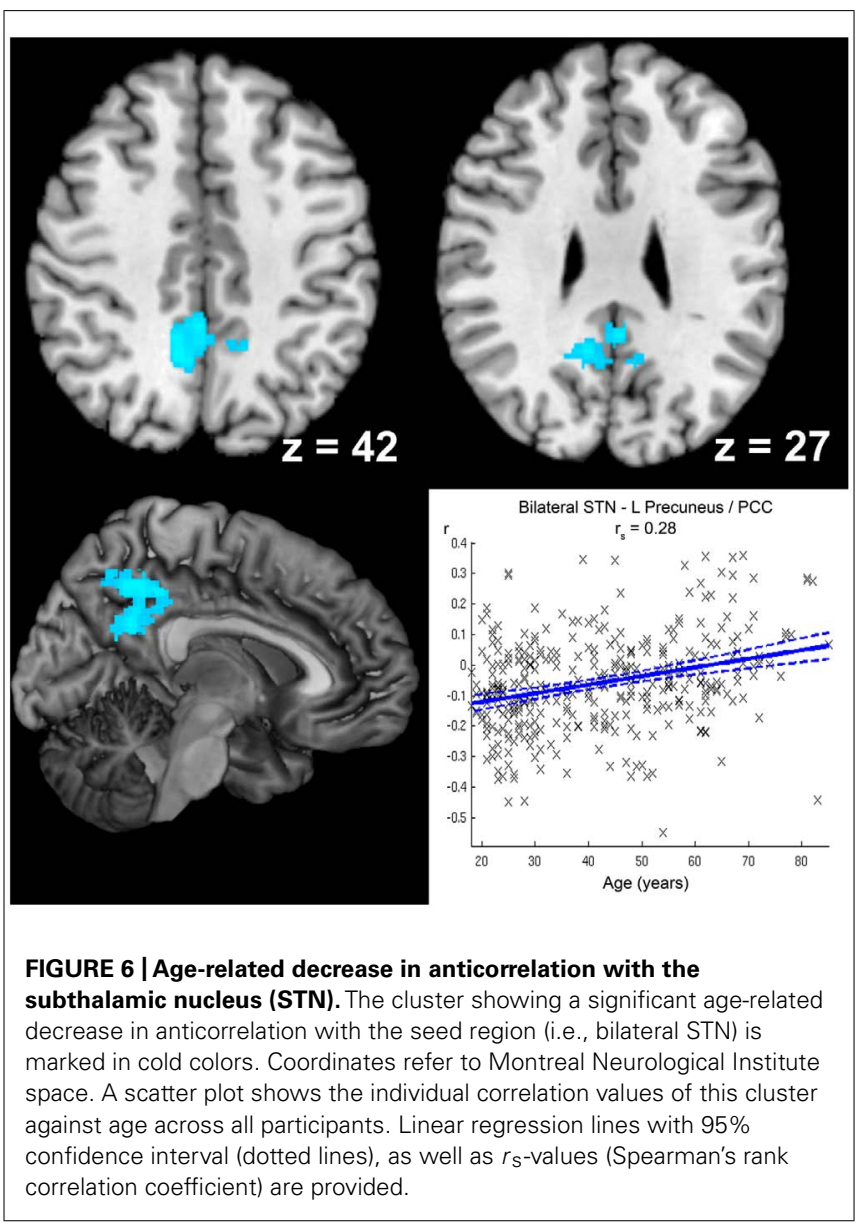

Like in the caudate nucleus, less interference by the STN might enhance cognitive processing in a compensatory manner. Selection of relevant sensory information for integration in the also affected parietal thalamic subregion (Eckert et al., 2012) could similarly be interpreted as another cognitive process that is shut off from STN influences. However, as an alternative hypothesis, a certain amount of influence by the STN on the parietal thalamic subregion might be necessary for sensory filtering. Hence, the age-related decline in FC between both regions could also contribute to changes in sensorimotor performance in the elderly (Seidler et al., 2010).

Our data also revealed an age-related reduction of STN FC with the posterior and central insula. A hierarchical model of the human insula suggests that sensorimotor information initially reaches the insula at its posterior part and is subsequently integrated with emotional and cognitive evaluations in the anterior insula (Craig, 2009; Kurth et al., 2010b). Although these mechanisms are not yet fully understood, it seems very likely that the insula has a superordinate role in interactions between motor, cognitive, and affective functions (Kurth et al., 2010b; Kelly et al., 2012).

Age-related increase in positive STN FC found in the sensorimotor cortex of right face and neck also supports the notion of an age-related shift of the STN coupling away from cognitivemotor toward basic-motor control. To our knowledge, there are no reports of age-related changes in facial motor function.

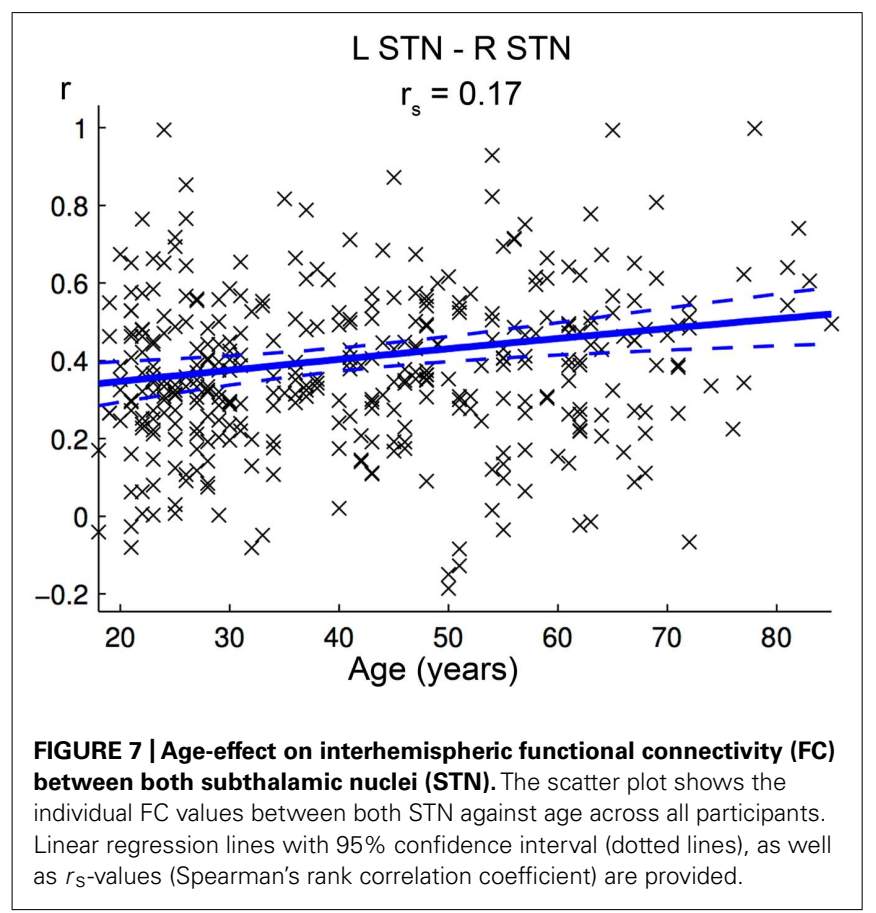

However, hypomimia is a typical symptom in PD patients. Since the prevalence of PD rises with age (de Lau and Breteler, 2006), and incidence of face and neck motor symptoms is known to increase with age (Szewczyk-Krolikowski et al., 2014), these symptoms could be the result of a combination of PD-related oscillatory hyperactivity of the STN (Kuhn etal., 2009) and the observed increased connectivity between STN and corresponding areas in M1.

If the alternative hypothesis is true and motor related activity in sensorimotor cortex triggers STN activity, this network could present a regulatory circuit in which motor activity at the same time stimulates the STN to increase response conservativeness in the motor network. The physiological value of this kind of regulation could lie in preventing initiation of too many new motor programs as long as previous actions are still in the phase of execution. In light of an age-related decline of sensorimotor abilities (Seidler et al., 2010), an increasing need for such a network and, therefore, an enhancement of its connections would seem plausible.

Remarkably, all these age-related changes of positive STN FC were found unilaterally. Bilateral insular clusters were identified only when adopting a more liberal cluster-level threshold $(p<0.1)$. While the lateralization of age-related changes in STN FC with M1 remains especially ambiguous, BOLD-measured activation induced by motor activity was reported to be higher for left, relative to right, basal ganglia in right-handers, regardless of the hand used (Scholz et al., 2000). Although the handedness of participants was not assessed in this study, preponderance of righthandedness is most likely. Potentially, the connection between STN and left putamen is also assigned to motor functions showing leftlateralization at the basal-ganglia level. This could explain why age-related changes in this connection reach detectability only in the left hemisphere. 
On the other hand, the right (versus left) striatum appears to be of particular importance for some cognitive processes like active learning (as compared with observational learning), with the caudate nucleus being especially involved in the evaluation of one's own behavioral outcome (Bellebaum et al., 2012). This underlines the importance of the right caudate nucleus for cognition and our notion of an age-related shift of the STN coupling away from cognitive functions. More specifically, this pattern could mean that the connection between STN and right caudate nucleus is also dedicated to active learning. If the STN drives the caudate nucleus, this might reflect compensating for an age-associated decline in motor learning (Seidler, 2006) by releasing learning processes in the caudate nucleus from interference by the STN. However, if the alternative hypothesis is true and the caudate nucleus drives the STN, learning processes in the caudate nucleus might co-activate the STN for suppression of potentially distracting motor programs. Decline of this connection could therefore be interpreted as a possible cause for the aforementioned age-related worsening of motor learning.

Positive FC between left and right STN increased significantly with age. To our knowledge, implications of such changes in STN interconnectivity for cognition and behavior have not yet been described in the literature. However, it seems possible that the above-mentioned functional segregation of the basal ganglia (right: focus on cognitive functions; left: focus on basic motor functions) does also apply to the subthalamic nuclei. Accordingly, aging would be associated with a decline in this segregation of higher level cognitive functions and lower level motor control, providing a possible neural substrate contributing to the worsening of both motor and cognitive performance with age.

While the cognitive functions that were linked to age-related changes of positive STN FC are all associated with motor functions on a superordinate level, the following age-related changes of negative STN FC are essentially related to non-motor cognitive networks.

\section{AGE-RELATED CHANGES OF NEGATIVE FC OF THE STN}

For the precuneus and the posterior cingulate cortex, we found an age-related decrease in functional anticorrelation (i.e., the neural de-coupling became weaker with age). The posterior cingulate cortex is part of the so-called default-mode network (Leech and Sharp, 2013) and has been implicated in self-reflection (Northoff et al., 2006; Qin and Northoff, 2011; Schilbach et al., 2012). The precuneus has been associated with introspection, memory, and mental imagery (Cavanna and Trimble, 2006). In sum, both posterior medial cortex regions (posterior cingulate cortex and precuneus) subserve cognitive functions that are unrelated to motor functions. Hence, together with the above-mentioned finding that aging influences the functional integration of the STN in motor-related networks, we also observed a reduction of its segregation from non-motor-related networks. The reduced decoupling of such non-motor regions suggests a reduced inhibition of cognitive processing that is unrelated to STN-related motor control functions and might thus lead to increased interference in advanced age. Interestingly, older adults' brain activity seems to focus on precuneus and posterior cingulate cortex when thinking about self-relevant agendas (especially duties and obligations), while activity of medial prefrontal cortex in the same context declines with age (Northoff et al., 2006; Mitchell et al., 2009). Hence, it seems conceivable that older adults' response control (mediated by the STN) has a higher chance of getting disturbed by such contemplations, as compared with younger adults.

\section{FC CHANGES IN LIGHT OF MICROSTRUCTURAL NEURODEGENERATION}

Several factors might underlie the discussed age-related changes in STN FC. Accumulating evidence points toward a central role of blood vessels within neurodegenerative processes. These factors include age-related dysfunction of cerebral autoregulation, structural and functional alterations in cerebral blood vessels including amyloid deposition, neurovascular uncoupling due to astrocyte endfeet retraction, changes in insulin/insulin-like growth factor-1 signaling, and secondary white matter changes (for a review, see Popa-Wagner et al., 2013). Due to high and variable energy demands, neurons are strongly reliant on adequate arterial inflow of oxygen and glucose, while, at the same time, they are highly vulnerable to hypoperfusion because of a very limited energy store (Gouix et al., 2014). An interrelationship between degenerative neuronal degradation and the observed age-related FC changes seems likely. However, current imaging techniques cannot discriminate between FC changes caused primarily by neuronal degradation and those which develop secondarily as adaptive mechanisms.

\section{LIMITATIONS AND FUTURE DIRECTIONS}

Our analysis has the typical limitation of a cross-sectional approach, which necessarily conflates age and cohort effects. Furthermore, although all participants of our analysis were without any record of neurological or psychiatric disorder, the efficiency of this screening at the three sites could have been different. Therefore, sporadic inclusion of participants with sub-clinical cognitive impairments cannot be completely excluded.

Finally, no behavioral data could be obtained from our participants, which would have been useful for testing the presumed cognitive and behavioral effects of the observed age-related FC changes. In future research, it would be desirable to combine the assessment of motor performance with the acquisition of RS-FC data in the same individuals to be able to directly test specific brain-behavior relationships.

\section{CONCLUSION}

Our examination of the global STN FC at "rest" demonstrated a positively coupled network comprising putamen, caudate nucleus, globus pallidus, thalamus, central and posterior insula, anterior and midcingulate cortex, sensorimotor cortex, V1, midbrain, cerebellum and pons. Advanced age was associated with a reduced coupling of the STN with cognitive motor control regions (caudate nucleus, thalamus, and central/posterior insula), increased coupling with lower level motor control regions (sensorimotor cortex and putamen), and reduced anti-correlation with non-motor regions (precuneus and posterior cingulate cortex).

Taken together, these connectivity changes suggest a shift of the STN's network involvement from more cognitive network coupling (caudate nucleus) toward more motor-related network coupling (putamen, M1). This connectivity changes could potentially 
provide a compensatory mechanism for the prevention or mitigation of the known degradation of motor performance with age. Apparently, older adults release superordinate motor functions (action selection; motor learning) in the caudate nucleus from interference by the STN and at the same time increase the influence of the STN on the putamen's and M1's basic motor functions toward more response conservativeness. Furthermore, the reduced de-coupling of non-motor-related brain areas might indicate a potential cause for age-related decline in motor performance: STN-mediated response control in older adults could thus become more prone to interference from self-relevant but action-irrelevant cognitive processing. In sum, our results suggest that aging affects the functional integration of the STN in motor-related networks and its segregation from non-motor-related networks.

\section{AUTHOR CONTRIBUTIONS}

Simon B. Eickhoff, Robert Langner, Felix Hoffstaedter, and Christian Mathys substantially contributed to the conception and design of the work. All authors substantially contributed to the analysis and interpretation of data for the work. Christian Mathys, Felix Hoffstaedter, and Robert Langner drafted the work. Julian Caspers, Svenja Caspers, Martin Südmeyer, Christian Grefkes, and Simon B. Eickhoff revised the work critically for important intellectual content. All authors finally approved the manuscript version to be published. All authors agree to be accountable for all aspects of the work in ensuring that questions related to the accuracy or integrity of any part of the work are appropriately investigated and resolved.

\section{ACKNOWLEDGMENTS}

This study was supported by the NIMH (R01-MH074457-01A1; Simon B. Eickhoff), the Initiative and Networking Fund of the Helmholtz Association within the Helmholtz Alliance on Systems Biology (Human Brain Model; Simon B. Eickhoff), and the German Research Foundation (DFG; EI 816/4-1, Simon B. Eickhoff; and LA 3071/3-1, Robert Langner, Simon B. Eickhoff). Christian Grefkes is supported by the German Research Foundation (DFG, GR 3285/2-1; GR3285/5-1 KFO219-TP8).

\section{REFERENCES}

Alexander, G. E., and Crutcher, M. D. (1990). Functional architecture of basal ganglia circuits: neural substrates of parallel processing. Trends Neurosci. 13, 266-271. doi: 10.1016/0166-2236(90)90107-L

Amunts, K., Kedo, O., Kindler, M., Pieperhoff, P., Mohlberg, H., Shah, N. J., et al. (2005). Cytoarchitectonic mapping of the human amygdala, hippocampal region and entorhinal cortex: intersubject variability and probability maps. Anat. Embryol. 210, 343-352. doi: 10.1007/s00429-0050025-5

Amunts, K., Malikovic, A., Mohlberg, H., Schormann, T., and Zilles, K. (2000). Brodmann's areas 17 and 18 brought into stereotaxic space-where and how variable? Neuroimage 11, 66-84. doi: 10.1006/nimg.1999.0516

Amunts, K., Schleicher, A., Burgel, U., Mohlberg, H., Uylings, H. B., and Zilles, K. (1999). Broca's region revisited: cytoarchitecture and intersubject variability. J. Comp. Neurol. 412, 319-341. doi: 10.1002/(SICI)10969861(19990920)412:2<319::AID-CNE10>3.0.CO;2-7

Amunts, K., Weiss, P. H., Mohlberg, H., Pieperhoff, P., Eickhoff, S., Gurd, J. M., etal. (2004). Analysis of neural mechanisms underlying verbal fluency in cytoarchitectonically defined stereotaxic space - the roles of Brodmann areas 44 and 45. Neuroimage 22, 42-56. doi: 10.1016/j.neuroimage.2003. 12.031
Aravamuthan, B. R., Muthusamy, K. A., Stein, J. F., Aziz, T. Z., and JohansenBerg, H. (2007). Topography of cortical and subcortical connections of the human pedunculopontine and subthalamic nuclei. Neuroimage 37, 694-705. doi: 10.1016/j.neuroimage.2007.05.050

Aron, A. R., Behrens, T. E., Smith, S., Frank, M. J., and Poldrack, R. A. (2007). Triangulating a cognitive control network using diffusion-weighted magnetic resonance imaging (MRI) and functional MRI. J. Neurosci. 27, 3743-3752. doi: 10.1523/JNEUROSCI.0519-07.2007

Ashburner, J., and Friston, K. J. (2005). Unified segmentation. Neuroimage 26, 839-851. doi: 10.1016/j.neuroimage.2005.02.018

Baudrexel, S., Witte, T., Seifried, C., Von Wegner, F., Beissner, F., Klein, J. C., et al. (2011). Resting state fMRI reveals increased subthalamic nucleus-motor cortex connectivity in Parkinson's disease. Neuroimage 55, 1728-1738. doi: 10.1016/j.neuroimage.2011.01.017

Beckmann, M., Johansen-Berg, H., and Rushworth, M. F. (2009). Connectivitybased parcellation of human cingulate cortex and its relation to functional specialization. J. Neurosci. 29, 1175-1190. doi: 10.1523/JNEUROSCI.332808.2009

Behrens, T. E., Johansen-Berg, H., Woolrich, M. W., Smith, S. M., WheelerKingshott, C. A., Boulby, P. A., et al. (2003). Non-invasive mapping of connections between human thalamus and cortex using diffusion imaging. Nat. Neurosci. 6, 750-757. doi: 10.1038/nn1075

Bellebaum, C., Jokisch, D., Gizewski, E. R., Forsting, M., and Daum, I. (2012). The neural coding of expected and unexpected monetary performance outcomes: dissociations between active and observational learning. Behav. Brain Res. 227, 241-251. doi: 10.1016/j.bbr.2011.10.042

Biswal, B. B., Mennes, M., Zuo, X. N., Gohel, S., Kelly, C., Smith, S. M., et al. (2010). Toward discovery science of human brain function. Proc. Natl. Acad. Sci. U.S.A. 107, 4734-4739. doi: 10.1073/pnas.0911855107

Biswal, B., Yetkin, F. Z., Haughton, V. M., and Hyde, J. S. (1995). Functional connectivity in the motor cortex of resting human brain using echo-planar MRI. Magn. Reson. Med. 34, 537-541. doi: 10.1002/mrm.1910340409

Brittain, J. S., Watkins, K. E., Joundi, R. A., Ray, N. J., Holland, P., Green, A. L., et al. (2012). A role for the subthalamic nucleus in response inhibition during conflict. J. Neurosci. 32, 13396-13401. doi: 10.1523/JNEUROSCI.2259-12.2012

Brown, P., and Marsden, C. D. (1998). What do the basal ganglia do? Lancet 351, 1801-1804. doi: 10.1016/S0140-6736(97)11225-9

Brunenberg, E. J., Moeskops, P., Backes, W. H., Pollo, C., Cammoun, L., Vilanova, A., etal. (2012). Structural and resting state functional connectivity of the subthalamic nucleus: identification of motor STN parts and the hyperdirect pathway. PLOS ONE 7:e39061. doi: 10.1371/journal.pone. 0039061

Caspers, S., Eickhoff, S. B., Geyer, S., Scheperjans, F., Mohlberg, H., Zilles, K., et al. (2008). The human inferior parietal lobule in stereotaxic space. Brain Struct. Funct. 212, 481-495. doi: 10.1007/s00429-008-0195-Z

Caspers, S., Geyer, S., Schleicher, A., Mohlberg, H., Amunts, K., and Zilles, K. (2006). The human inferior parietal cortex: cytoarchitectonic parcellation and interindividual variability. Neuroimage 33, 430-448. doi: 10.1016/j.neuroimage.2006.06.054

Cavanna, A. E., and Trimble, M. R. (2006). The precuneus: a review of its functional anatomy and behavioural correlates. Brain 129, 564-583. doi: 10.1093/brain/awl004

Cieslik, E. C., Zilles, K., Caspers, S., Roski, C., Kellermann, T. S., Jakobs, O., et al. (2013). Is there "one" DLPFC in cognitive action control? Evidence for heterogeneity from co-activation-based parcellation. Cereb. Cortex 23, 2677-2689. doi: $10.1093 /$ cercor/bhs 256

Cohen, J. (1988). Statistical Power Analysis for the Behavioral Sciences, 2nd Edn. Hillsdale, NJ: LawrenceErlbaum Associates.

Craig, A. D. (2009). How do you feel - now? The anterior insula and human awareness. Nat. Rev. Neurosci. 10, 59-70. doi: 10.1038/nrn2555

de Lau, L. M., and Breteler, M. M. (2006). Epidemiology of Parkinson's disease. Lancet Neurol. 5, 525-535. doi: 10.1016/S1474-4422(06)70471-9

Devinsky, O., Morrell, M. J., and Vogt, B. A. (1995). Contributions of anterior cingulate cortex to behaviour. Brain 118, 279-306. doi: 10.1093/brain/118.1.279

Diedrichsen, J., Balsters, J. H., Flavell, J., Cussans, E., and Ramnani, N. (2009). A probabilistic MR atlas of the human cerebellum. Neuroimage 46, 39-46. doi: 10.1016/j.neuroimage.2009.01.045

Eckert, U., Metzger, C. D., Buchmann, J. E., Kaufmann, J., Osoba, A., Li, M., etal. (2012). Preferential networks of the mediodorsal nucleus and 
centromedian-parafascicular complex of the thalamus - a DTI tractography study. Hum. Brain Mapp. 33, 2627-2637. doi: 10.1002/hbm.21389

Eickhoff, S. B., Amunts, K., Mohlberg, H., and Zilles, K. (2006a). The human parietal operculum. II. Stereotaxic maps and correlation with functional imaging results. Cereb. Cortex 16, 268-279. doi: 10.1093/cercor/bhi106

Eickhoff, S. B., Schleicher, A., Zilles, K., and Amunts, K. (2006b). The human parietal operculum. I. Cytoarchitectonic mapping of subdivisions. Cereb. Cortex 16, 254-267. doi: 10.1093/cercor/bhi105

Eickhoff, S. B., Paus, T., Caspers, S., Grosbras, M. H., Evans, A. C., Zilles, K., et al. (2007). Assignment of functional activations to probabilistic cytoarchitectonic areas revisited. Neuroimage 36, 511-521. doi: 10.1016/j.neuroimage.2007.03.060

Fox, M. D., and Raichle, M. E. (2007). Spontaneous fluctuations in brain activity observed with functional magnetic resonance imaging. Nat. Rev. Neurosci. 8, 700-711. doi: 10.1038/nrn2201

Frank, M. J. (2006). Hold your horses: a dynamic computational role for the subthalamic nucleus in decision making. Neural Netw. 19, 1120-1136. doi: 10.1016/j.neunet.2006.03.006

Frank, M. J., Samanta, J., Moustafa, A. A., and Sherman, S. J. (2007). Hold your horses: impulsivity, deep brain stimulation, and medication in parkinsonism. Science 318, 1309-1312. doi: 10.1126/science.1146157

Geyer, S. (2004). The microstructural border between the motor and the cognitive domain in the human cerebral cortex. Adv. Anat. Embryol. Cell Biol. 174, I-VIII, 1-89. doi: 10.1007/978-3-642-18910-4_1

Geyer, S., Ledberg, A., Schleicher, A., Kinomura, S., Schormann, T., Burgel, U., et al. (1996). Two different areas within the primary motor cortex of man. Nature 382, 805-807. doi: 10.1038/382805a0

Geyer, S., Schleicher, A., and Zilles, K. (1999). Areas 3a, 3b, and 1 of human primary somatosensory cortex. Neuroimage 10, 63-83. doi: 10.1006/nimg. 1999.0440

Geyer, S., Schormann, T., Mohlberg, H., and Zilles, K. (2000). Areas 3a, 3b, and 1 of human primary somatosensory cortex. Part 2. Spatial normalization to standard anatomical space. Neuroimage 11, 684-696. doi: 10.1006/nimg.2000.0548

Goble, D. J., Coxon, J. P., Van Impe, A., Geurts, M., Van Hecke, W., Sunaert, S., et al. (2012). The neural basis of central proprioceptive processing in older versus younger adults: an important sensory role for right putamen. Hum. Brain Mapp. 33, 895-908. doi: 10.1002/hbm.21257

Gouix, E., Buisson, A., Nieoullon, A., Kerkerian-Le Goff, L., Tauskela, J. S., Blondeau, N., et al. (2014). Oxygen glucose deprivation-induced astrocyte dysfunction provokes neuronal death through oxidative stress. Pharmacol. Res. 11, 8-17. doi: 10.1016/j.phrs.2014.06.002

Grady, C. L. (2005). "Functional connectivity during memory tasks in healthy aging and dementia," in Cognitive Neuroscience of Aging: Linking Cognitive and Cerebral Aging, eds R. Cabeza, L. Nyberg, and D. C. Park (Oxford: Oxford University Press), 286-308.

Grahn, J. A., Parkinson, J. A., and Owen, A. M. (2008). The cognitive functions of the caudate nucleus. Prog. Neurobiol. 86, 141-155. doi 10.1016/j.pneurobio.2008.09.004

Graybiel, A. M. (2005). The basal ganglia: learning new tricks and loving it. Curr. Opin. Neurobiol. 15, 638-644. doi: 10.1016/j.conb.2005.10.006

Grefkes, C., Geyer, S., Schormann, T., Roland, P., and Zilles, K. (2001). Human somatosensory area 2: observer-independent cytoarchitectonic mapping, interindividual variability, and population map. Neuroimage 14, 617-631. doi: 10.1006/nimg.2001.0858

Greicius, M. D., Krasnow, B., Reiss, A. L., and Menon, V. (2003). Functional connectivity in the resting brain: a network analysis of the default mode hypothesis Proc. Natl. Acad. Sci. U.S.A 100, 253-258. doi: 10.1073/pnas.0135058100

Hamani, C., Saint-Cyr, J. A., Fraser, J., Kaplitt, M., and Lozano, A. M. (2004). The subthalamic nucleus in the context of movement disorders. Brain 127, 4-20. doi: 10.1093/brain/awh029

Harada, T., Miyai, I., Suzuki, M., and Kubota, K. (2009). Gait capacity affects cortical activation patterns related to speed control in the elderly. Exp. Brain Res. 193, 445-454. doi: 10.1007/s00221-008-1643-y

Hardwick, R. M., Rottschy, C., Miall, R. C., and Eickhoff, S. B. (2013). A quantitative meta-analysis and review of motor learning in the human brain. Neuroimage 67, 283-297. doi: 10.1016/j.neuroimage.2012.11.020

Heuninckx, S., Wenderoth, N., Debaere, F., Peeters, R., and Swinnen, S. P. (2005) Neural basis of aging: the penetration of cognition into action control. J. Neurosci. 25, 6787-6796. doi: 10.1523/JNEUROSCI.1263-05.2005
Hoffstaedter, F., Grefkes, C., Caspers, S., Roski, C., Palomero-Gallagher, N., Laird, A. R., et al. (2013a). The role of anterior midcingulate cortex in cognitive motor control: evidence from functional connectivity analyses. Hum. Brain Mapp. 35, 2741-2753. doi: 10.1002/hbm.22363

Hoffstaedter, F., Grefkes, C., Zilles, K., and Eickhoff, S. B. (2013b). The "what" and "when" of self-initiated movements. Cereb. Cortex 23, 520-530. doi: $10.1093 /$ cercor/bhr391

Hoffstaedter, F., Grefkes, C., Roski, C., Caspers, S., Zilles, K., and Eickhoff, S. B. (2014). Age-related decrease of functional connectivity additional to gray matter atrophy in a network for movement initiation. Brain Struct. Funct. doi: 10.1007/s00429-013-0696-2 [Epub ahead of print].

Jakobs, O., Langner, R., Caspers, S., Roski, C., Cieslik, E. C., Zilles, K., et al. (2012). Across-study and within-subject functional connectivity of a right temporoparietal junction subregion involved in stimulus-context integration. Neuroimage 60, 2389-2398. doi: 10.1016/j.neuroimage.2012.02.037

Jueptner, M., Stephan, K. M., Frith, C. D., Brooks, D. J., Frackowiak, R. S., and Passingham, R. E. (1997). Anatomy of motor learning. I. Frontal cortex and attention to action. J. Neurophysiol. 77, 1313-1324.

Kelly, A. M., Di Martino, A., Uddin, L. Q., Shehzad, Z., Gee, D. G., Reiss, P. T., et al. (2009). Development of anterior cingulate functional connectivity from late childhood to early adulthood. Cereb. Cortex 19, 640-657. doi: 10.1093/cercor/bhn117

Kelly, C., Toro, R., Di Martino, A., Cox, C. L., Bellec, P., Castellanos, F. X., et al. (2012). A convergent functional architecture of the insula emerges across imaging modalities. Neuroimage 61, 1129-1142. doi: 10.1016/j.neuroimage.2012.03.021

Keuken, M. C., Bazin, P. L., Schafer, A., Neumann, J., Turner, R., and Forstmann, B. U. (2013). Ultra-high 7T MRI of structural age-related changes of the subthalamic nucleus. J. Neurosci. 33, 4896-4900. doi: 10.1523/JNEUROSCI.324112.2013

Kraft, E., Chen, A. W., Flaherty, A. W., Blood, A. J., Kwong, K. K., and Jenkins, B. G. (2007). The role of the basal ganglia in bimanual coordination. Brain Res. 1151, 62-73. doi: 10.1016/j.brainres.2007.01.142

Kuhn, A. A., Tsui, A., Aziz, T., Ray, N., Brucke, C., Kupsch, A., et al. (2009). Pathological synchronisation in the subthalamic nucleus of patients with Parkinson's disease relates to both bradykinesia and rigidity. Exp. Neurol. 215, 380-387. doi: 10.1016/j.expneurol.2008.11.008

Kurth, F., Eickhoff, S. B., Schleicher, A., Hoemke, L., Zilles, K., and Amunts, K. (2010a). Cytoarchitecture and probabilistic maps of the human posterior insular cortex. Cereb. Cortex 20, 1448-1461. doi: 10.1093/cercor/ bhp208

Kurth, F., Zilles, K., Fox, P. T., Laird, A. R., and Eickhoff, S. B. (2010b). A link between the systems: functional differentiation and integration within the human insula revealed by meta-analysis. Brain Struct. Funct. 214, 519-534. doi: 10.1007/s00429010-0255-z

Lambert, C., Zrinzo, L., Nagy, Z., Lutti, A., Hariz, M., Foltynie, T., et al. (2012). Confirmation of functional zones within the human subthalamic nucleus: patterns of connectivity and sub-parcellation using diffusion weighted imaging. Neuroimage 60, 83-94. doi: 10.1016/j.neuroimage.2011.11.082

Langner, R., Cieslik, E. C., Behrwind, S. D., Roski, C., Caspers, S., Amunts, K., et al. (2014). Aging and response conflict solution: behavioural and functional connectivity changes. Brain Struct. Funct. doi: 10.1007/s00429-014-0758-0 [Epub ahead of print].

Langner, R., Kellermann, T., Boers, F., Sturm, W., Willmes, K., and Eickhoff, S. B. (2011). Modality-specific perceptual expectations selectively modulate baseline activity in auditory, somatosensory, and visual cortices. Cereb. Cortex 21, 28502862. doi: $10.1093 /$ cercor/bhr083

Langner, R., Kellermann, T., Eickhoff, S. B., Boers, F., Chatterjee, A., Willmes, K., et al. (2012). Staying responsive to the world: modality-specific and -nonspecific contributions to speeded auditory, tactile, and visual stimulus detection. Hum. Brain Mapp. 33, 398-418. doi: 10.1002/hbm.21220

Lee, T. M., Zhang, J. X., Chan, C. C., Yuen, K. S., Chu, L. W., Cheung, R. T., et al. (2006). Age-related differences in response regulation as revealed by functional MRI. Brain Res. 1076, 171-176. doi: 10.1016/j.brainres.2005. 12.124

Leech, R., and Sharp, D. J. (2013). The role of the posterior cingulate cortex in cognition and disease. Brain 137, 12-32. doi: 10.1093/brain/awt162

Le Jeune, F., Peron, J., Grandjean, D., Drapier, S., Haegelen, C., Garin, E., et al. (2010). Subthalamic nucleus stimulation affects limbic and associative circuits: a 
PET study. Eur. J. Nucl. Med. Mol. Imaging 37, 1512-1520. doi: 10.1007/s00259010-1436-y

Limousin, P., Pollak, P., Benazzouz, A., Hoffmann, D., Le Bas, J. F., Broussolle, E., et al. (1995). Effect of parkinsonian signs and symptoms of bilateral subthalamic nucleus stimulation. Lancet 345, 91-95. doi: 10.1016/S0140-6736(95) 90062-4

Madden, D. J., Costello, M. C., Dennis, N. A., Davis, S. W., Shepler, A. M., Spaniol, J., et al. (2010). Adult age differences in functional connectivity during executive control. Neuroimage 52, 643-657. doi: 10.1016/j.neuroimage.2010. 04.249

Manes, J. L., Parkinson, A. L., Larson, C. R., Greenlee, J. D., Eickhoff, S. B., Corcos, D. M., et al. (2013). Connectivity of the subthalamic nucleus and globus pallidus pars interna to regions within the speech network: a meta-analytic connectivity study. Hum. Brain Mapp. 35. doi: 10.1002/hbm.22417

Mansfield, E. L., Karayanidis, F., Jamadar, S., Heathcote, A., and Forstmann, B. U. (2011). Adjustments of response threshold during task switching: a model-based functional magnetic resonance imaging study. J. Neurosci. 31, 14688-14692. doi: 10.1523/JNEUROSCI.2390-11.2011

Margulies, D. S., Kelly, A. M., Uddin, L. Q., Biswal, B. B., Castellanos, F. X., and Milham, M. P. (2007). Mapping the functional connectivity of anterior cingulate cortex. Neuroimage 37, 579-588. doi: 10.1016/j.neuroimage.2007.05.019

Meier, J. D., Aflalo, T. N., Kastner, S., and Graziano, M. S. (2008). Complex organization of human primary motor cortex: a high-resolution fMRI study. J. Neurophysiol. 100, 1800-1812. doi: 10.1152/jn.90531.2008

Meissner, W., Leblois, A., Hansel, D., Bioulac, B., Gross, C. E., Benazzouz, A., et al. (2005). Subthalamic high frequency stimulation resets subthalamic firing and reduces abnormal oscillations. Brain 128, 2372-2382. doi: 10.1093/brain/awh616

Mitchell, K. J., Raye, C. L., Ebner, N. C., Tubridy, S. M., Frankel, H., and Johnson, M. K. (2009). Age-group differences in medial cortex activity associated with thinking about self-relevant agendas. Psychol. Aging 24, 438-449. doi: 10.1037/a0015181

Nambu, A., Tokuno, H., and Takada, M. (2002). Functional significance of the cortico-subthalamo-pallidal 'hyperdirect' pathway. Neurosci. Res. 43, 111-117. doi: 10.1016/S0168-0102(02)00027-5

Noble, J. W., Eng, J. J., Kokotilo, K. J., and Boyd, L. A. (2011). Aging effects on the control of grip force magnitude: an fMRI study. Exp. Gerontol. 46, 453-461. doi: 10.1016/j.exger.2011.01.004

Northoff, G., Heinzel, A., De Greck, M., Bermpohl, F., Dobrowolny, H., and Panksepp, J. (2006). Self-referential processing in our brain - a metaanalysis of imaging studies on the self. Neuroimage 31, 440-457. doi: 10.1016/j.neuroimage.2005.12.002

Packard, M. G., and Knowlton, B. J. (2002). Learning and memory functions of the Basal Ganglia. Annu. Rev. Neurosci. 25, 563-593. doi: 10.1146/annurev.neuro.25112701.142937

Pollok, B., Kamp, D., Butz, M., Wojtecki, L., Timmermann, L., Sudmeyer, M., et al. (2013). Increased SMA-M1 coherence in Parkinson's disease - pathophysiology or compensation? Exp. Neurol. 247, 178-181. doi: 10.1016/j.expneurol.2013.04.013

Popa-Wagner, A., Buga, A. M., Popescu, B., and Muresanu, D. (2013). Vascular cognitive impairment, dementia, aging and energy demand. A vicious cycle. $J$. Neural Transm. doi: 10.1007/s00702-013-1129-3 [Epub ahead of print].

Qin, P., and Northoff, G. (2011). How is our self related to midline regions and the default-mode network? Neuroimage 57, 1221-1233. doi: 10.1016/j.neuroimage.2011.05.028

Rorden, C., and Brett, M. (2000). Stereotaxic display of brain lesions. Behav. Neurol. 12, 191-200. doi: 10.1155/2000/421719

Rottschy, C., Eickhoff, S. B., Schleicher, A., Mohlberg, H., Kujovic, M., Zilles, K., et al. (2007). Ventral visual cortex in humans: cytoarchitectonic mapping of two extrastriate areas. Hum. Brain Mapp. 28, 1045-1059. doi: 10.1002/hbm.20348

Rubia, K., Overmeyer, S., Taylor, E., Brammer, M., Williams, S. C., Simmons, A., et al. (1999). Hypofrontality in attention deficit hyperactivity disorder during higher-order motor control: a study with functional MRI. Am. J. Psychiatry 156, 891-896. doi: 10.1176/appi.pn.2014.2a3

Satterthwaite, T. D., Elliott, M. A., Gerraty, R. T., Ruparel, K., Loughead, J., Calkins, M. E., et al. (2013a). An improved framework for confound regression and filtering for control of motion artifact in the preprocessing of resting-state functional connectivity data. Neuroimage 64, 240-256. doi: 10.1016/j.neuroimage.2012.08.052

Satterthwaite, T. D., Wolf, D. H., Ruparel, K., Erus, G., Elliott, M. A., Eickhoff, S. B., et al. (2013b). Heterogeneous impact of motion on fundamental patterns of developmental changes in functional connectivity during youth. Neuroimage 83, 45-57. doi: 10.1016/j.neuroimage.2013.06.045

Schafer, A., Forstmann, B. U., Neumann, J., Wharton, S., Mietke, A., Bowtell, R., et al. (2012). Direct visualization of the subthalamic nucleus and its iron distribution using high-resolution susceptibility mapping. Hum. Brain Mapp. 33, 2831-2842. doi: 10.1002/hbm.21404

Scheperjans, F., Eickhoff, S. B., Homke, L., Mohlberg, H., Hermann, K., Amunts, K., et al. (2008a). Probabilistic maps, morphometry, and variability of cytoarchitectonic areas in the human superior parietal cortex. Cereb. Cortex 18, 2141-2157. doi: $10.1093 /$ cercor/bhm 241

Scheperjans, F., Hermann, K., Eickhoff, S. B., Amunts, K., Schleicher, A., and Zilles, K. (2008b). Observer-independent cytoarchitectonic mapping of the human superior parietal cortex. Cereb. Cortex 18, 846-867. doi: 10.1093/cercor/bhm116 Schilbach, L., Bzdok, D., Timmermans, B., Fox, P. T., Laird, A. R., Vogeley, K., et al. (2012). Introspective minds: using ALE meta-analyses to study commonalities in the neural correlates of emotional processing, social \& unconstrained cognition. PLoS ONE 7:e30920. doi: 10.1371/journal.pone.0030920

Scholz, V. H., Flaherty, A. W., Kraft, E., Keltner, J. R., Kwong, K. K., Chen, Y. I., et al. (2000). Laterality, somatotopy and reproducibility of the basal ganglia and motor cortex during motor tasks. Brain Res. 879, 204-215. doi: 10.1016/S00068993(00)02749-9

Seidler, R. D. (2006). Differential effects of age on sequence learning and sensorimotor adaptation. Brain Res. Bull. 70, 337-346. doi: 10.1016/j.brainresbull.2006.06.008

Seidler, R. D., Bernard, J. A., Burutolu, T. B., Fling, B. W., Gordon, M. T., Gwin, J. T., et al. (2010). Motor control and aging: links to age-related brain structural, functional, and biochemical effects. Neurosci. Biobehav. Rev. 34, 721-733. doi: 10.1016/j.neubiorev.2009.10.005

Szewczyk-Krolikowski, K., Tomlinson, P., Nithi, K., Wade-Martins, R., Talbot, K., Ben-Shlomo, Y., et al. (2014). The influence of age and gender on motor and non-motor features of early Parkinson's disease: initial findings from the Oxford Parkinson Disease Center (OPDC) discovery cohort. Parkinsonism relat. disord. 20, 99-105. doi: 10.1016/j.parkreldis.2013.09.025

Temel, Y., Blokland, A., Steinbusch, H. W., and Visser-Vandewalle, V. (2005). The functional role of the subthalamic nucleus in cognitive and limbic circuits. Prog. Neurobiol. 76, 393-413. doi: 10.1016/j.pneurobio.2005.09.005

Tommasi, G., Krack, P., Fraix, V., and Pollak, P. (2012). Effects of varying subthalamic nucleus stimulation on apraxia of lid opening in Parkinson's disease. J. Neurol. 259, 1944-1950. doi: 10.1007/s00415-012-6447-0

Ward, N. S., Swayne, O. B., and Newton, J. M. (2008). Age-dependent changes in the neural correlates of force modulation: an fMRI study. Neurobiol. Aging 29, 1434-1446. doi: 10.1016/j.neurobiolaging.2007.04.017

Weintraub, D. B., and Zaghloul, K. A. (2013). The role of the subthalamic nucleus in cognition. Rev. Neurosci. 24, 125-138. doi: 10.1515/revneuro-2012-0075

Williams, Z. M., and Eskandar, E. N. (2006). Selective enhancement of associative learning by microstimulation of the anterior caudate. Nat. Neurosci. 9, 562-568. doi: $10.1038 / \mathrm{nn} 1662$

Zilles, K., and Amunts, K. (2010). Centenary of Brodmann's map - conception and fate. Nature reviews. Neuroscience 11, 139-145. doi: 10.1038/nrn2776

Conflict of Interest Statement: The authors declare that the research was conducted in the absence of any commercial or financial relationships that could be construed as a potential conflict of interest.

Received: 13 May 2014; accepted: 04 July 2014; published online: 23 July 2014. Citation: Mathys C, Hoffstaedter F, Caspers J, Caspers S, Südmeyer M, Grefkes C, Eickhoff SB and Langner $R$ (2014) An age-related shift of resting-state functional connectivity of the subthalamic nucleus: a potential mechanism for compensating motor performance decline in older adults. Front. Aging Neurosci. 6:178. doi: 10.3389/fnagi.2014.00178

This article was submitted to the journal of the Frontiers in Aging Neuroscience. Copyright $\odot 2014$ Mathys, Hoffstaedter, Caspers, Caspers, Südmeyer, Grefkes, Eickhoff and Langner. This is an open-access article distributed under the terms of the Creative Commons Attribution License (CC BY). The use, distribution or reproduction in other forums is permitted, provided the original author(s) or licensor are credited and that the original publication in this journal is cited, in accordance with accepted academic practice. No use, distribution or reproduction is permitted which does not comply with these terms. 